\title{
Yttrium Y 90-Edotreotide
}

National Cancer Institute

\section{Source}

National Cancer Institute. Yttrium Y 90-Edotreotide. NCI Thesaurus. Code C2601.

A radioconjug ate consisting of the octreotide derivative edotreotide labeled with yttrium 90 (Y-90) with potential radiotherapeutic uses. Similar to octreotide, yttrium Y 90edotreotide binds to somatostatin receptors (SST Rs), especially type 2 receptors, present on the cell membranes of many types of neuroendocrine tumor cells, delivering tissue-specific, beta-emitting nuclide Y-90-mediated cytotoxicity to SST R-positive cells. Yttrium Y 90-edotreotide is produced by substituting tyrosine for phenylalanine at the 3 position of the somatostatin analogue octreotide and chelating the substituted octreotide to Y-90 via dodecanetetraacetic acid (DOTA). 